\title{
Transcriptional control of circadian metabolic rhythms in the liver
}

\author{
S. Li \& J. D. Lin \\ Life Sciences Institute and Department of Cell \& Developmental Biology, University of Michigan, Ann Arbor, MI, USA
}

Diurnal metabolic rhythms add an important temporal dimension to metabolic homeostasis in mammals. Although it remains a challenge to untangle the intricate networks of crosstalk among the body clock, nutrient signalling and tissue metabolism, there is little doubt that the rhythmic nature of nutrient and energy metabolism is a central aspect of metabolic physiology. Disruption of the synchrony between clock and metabolism has been causally linked to diverse pathophysiological states. As such, restoring the rhythmicity of body physiology and therapeutic targeting directed at specific time windows during the day may have important implications in human health and medicine. In this review, we summarize recent findings on the integration of hepatic glucose metabolism and the body clock through a regulatory network centred on the PPAR $\gamma$ coactivator 1 (PGC-1) transcriptional coactivators. In addition, we discuss the transcriptional mechanisms underlying circadian control of the autophagy gene programme and autophagy in the liver.

Keywords: autophagy, circadian rhythm, clock, glucose, lipid, liver, metabolism, PGC-1, transcription

Date submitted 7 April 2015; date of final acceptance 25 April 2015

\section{Circadian Clock and Energy Metabolism}

The body clock controls many aspects of mammalian physiology, including the sleep-wake cycle, locomotor activity, hormone release and metabolism. These biological rhythms are entrained by the light-dark (LD) and feeding cycles. In mammals, the master clock is located in the suprachiasmatic nucleus (SCN) of the ventral hypothalamus. Light resets the phase of the central pacemaker, which in turn synchronizes clocks in peripheral tissues. Remarkably, peripheral oscillators can be uncoupled from the central clock in response to restricted feeding [1-3], underscoring the importance of nutritional signals in circadian timing in the peripheral tissues. Clock oscillators regulate diverse organismal functions, but also operate in a cell-autonomous manner. Tissue explants can maintain robust rhythmic patterns of gene expression over a period of days to weeks in culture dishes [4]. Further, individual fibroblasts harbour intrinsic rhythms that undergo synchronization in response to a variety of stimuli, such as brief serum shock and exposure to glucocorticoids or forskolin $[5,6]$.

Nutrient and energy metabolism is tightly integrated with the circadian timing system in mammals [7-9]. The concentrations of plasma metabolites such as amino acids and lipids, and circulating hormones, exhibit varying degrees of circadian rhythms. Recent metabolomic profiling of plasma in mice and humans revealed extensive diurnal oscillation [10-12], probably as a result of a combination of feeding cycles and endogenous metabolic rhythms. This daily ebb and flow of metabolites is accompanied by cyclic activation of major metabolic pathways in the liver, including gluconeogenesis, de novo lipogenesis, lipoprotein secretion, cholesterol

Correspondence to: J. Lin, PhD, 5437 Life Sciences Institute, University of Michigan, 210 Washtenaw Avenue, Ann Arbor, MI 48109, USA.

E-mail: jdlin@umich.edu biosynthesis and xenobiotic detoxification. At the molecular level, transcriptional profiling using microarray and RNA sequencing has revealed extensive rhythmic expression of hepatic genes throughout the LD cycles [13-17]. A significant number of these cycling genes encode enzymes involved in glucose, lipid, amino acid and mitochondrial oxidative metabolism. As such, many metabolic processes are restricted not only to certain tissues in the body but also during specific time periods during the day.

\section{$\mathbf{C C}_{\text {The integration of body clock and metabolism is }}$ orchestrated by reciprocal signalling between the clock oscillator and metabolic pathways. $)$}

Temporal restriction of metabolic functions may provide advantages for organisms as they anticipate and synchronize their physiology to the environment. It is therefore not entirely surprising that disruptions of circadian timing have been associated with diseases such as sleep disorders, cardiovascular disease, metabolic syndrome and certain cancers [18-23]. In the context of metabolic homeostasis, circadian misalignments have been shown to elevate blood glucose and insulin concentrations in healthy individuals, accompanied by reduced circulating leptin levels and increased arterial blood pressure [24,25]. Whole body insulin sensitivity and glucose tolerance were significantly impaired following disruption of normal sleep cycles. In mice, high-fat feeding directly impacts the body clock while meal timing by restricted feeding profoundly alters the course of diet-induced obesity and its associated metabolic disorders [26]. The metabolic phenotype of several clock gene mutants has been characterized. Clock mutant mice develop obesity and display characteristics of metabolic syndrome [27], whereas mice lacking Bmall, a heterodimer partner for Clock, have 
impaired glucose homeostasis and develop accelerated ageing $[28,29]$. Pancreas-specific inactivation of Bmal1 markedly impaired glucose-stimulated insulin secretion and resulted in hyperglycaemia in mice [30,31]. Deficiency of both Rev-erba and Rev-erb $\beta$ resulted in elevated blood glucose and plasma triglyceride levels and hepatic steatosis $[32,33]$. These observations show that different clock components exert distinct and tissue-specific effects on energy metabolism, underscoring the complex relationship between the molecular clock and metabolic pathways.

\section{Integration of Clock and Metabolism by the PGC-1 Coactivators}

The PPAR $\gamma$ coactivator 1 (PGC-1) family of transcriptional coactivators includes PGC- $1 \alpha$, PGC- $1 \beta$ and PGC-related coactivator $[34,35]$. PGC-1 $\alpha$ expression is highly inducible in brown fat by cold exposure [36] in the liver following starvation $[37,38]$ and in skeletal muscle in response to physical exercise $[39,40]$. Hepatic $P G C-1 \beta$ expression is responsive to dietary intake of saturated fats [41]. In many cell types, PGC- $1 \alpha$ and PGC- $1 \beta$ stimulate the expression of mitochondrial gene programmes encoded by both nuclear and mitochondrial genomes. PGC-1 $\alpha$ deficiency leads to impaired mitochondrial oxidative metabolism in several tissues, including the brain, heart, skeletal muscle, brown fat and the liver [42]. Similar defects of mitochondrial gene expression and respiratory function were also observed in $P G C-1 \beta$ null mouse tissues [43], suggesting that they play non-redundant roles in maintaining mitochondrial oxidative metabolism. A remarkable feature of PGC-1 action is that they coordinate diverse aspects of metabolic adaptation in tissues in response to nutritional and hormonal cues. For example, mice lacking PGC-1 $\alpha$ exhibited impaired cold-induced thermogenesis and exercise endurance and had aberrant induction of hepatic starvation response. Beyond mitochondrial metabolism, PGC-1 coactivators also regulate diverse biological processes in a cell type-specific manner. PGC- $1 \alpha$ stimulates hepatic gluconeogenesis [38] and oxidative muscle fibre formation [44], whereas PGC-1 $\beta$ promotes lipoprotein secretion $[41,45]$ and bone homeostasis [46].

Previous studies have shown that the expression of $P G C-1 \alpha$ and $P G C-1 \beta$ exhibits strong diurnal rhythms in the liver and skeletal muscle $[47,48]$. PGC- $1 \alpha$ induces the expression of several core clock genes, particularly Bmal1, Clock, Per2 and Rev-erb $\alpha$, in a cell-autonomous manner. The induction of clock gene expression is at least in part mediated through its coactivation of orphan nuclear receptor $\operatorname{ROR} \alpha$ (Figure 1). The PGC- $1 \alpha /$ ROR and Rev-erb $\alpha /$ HDAC transcriptional complexes appear to play an antagonistic role in the transcriptional regulation of Bmall expression [49,50]. The physiological role of PGC- $1 \alpha$ in normal clock function was supported by significant impairments of diurnal rhythms of locomotor activity, body temperature and metabolic rate in PGC-1 $\alpha$ null mice. Similarly, mice lacking PGC-1 $\beta$ also display abnormal diurnal locomotor activity patterns [51]. These results strongly implicate the PGC-1 coactivators as a nodal point in integrating energy metabolism and the body clock.

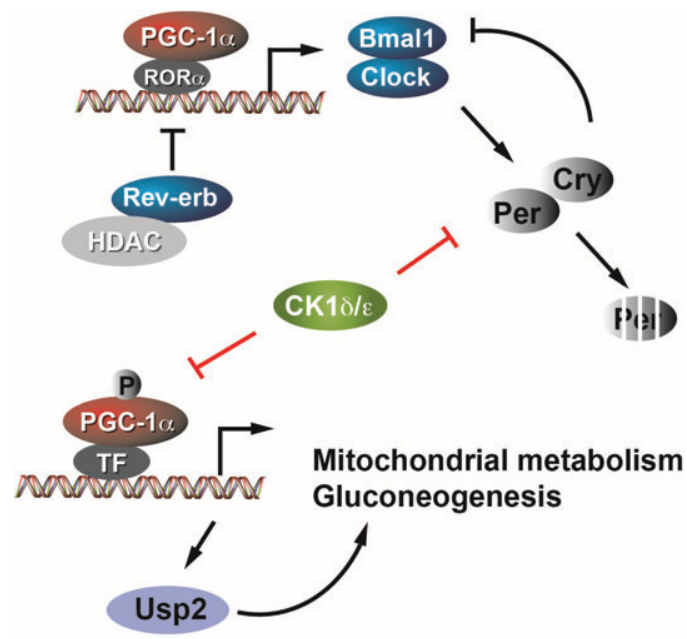

Figure 1. Integration of clock and hepatic metabolism by PGC- $1 \alpha$. PGC- $1 \alpha$ coordinately regulates clock and metabolic gene expression through coactivation of its transcriptional partners. PGC-1 $\alpha$ is reciprocally modulated by the biological clock through CK1 $\delta$-mediated phosphorylation. PGC-1, PPAR $\gamma$ coactivator 1 ; CK1 $\delta$, casein kinase $1 \delta$.

\section{Circadian Regulation of Hepatic Gluconeogenesis by PGC- $1 \alpha$}

At the molecular level, the PGC-1 coactivators exert their effects through physical interaction with transcription factors and chromatin-remodelling complexes. Both PGC- $1 \alpha$ and PGC- $1 \beta$ interact with nuclear respiratory factor 1 (NRF1), oestrogen receptor-related receptor $\alpha(\mathrm{ERR} \alpha)$ and $\operatorname{ERR} \gamma$ to regulate mitochondrial biogenesis $[34,35]$. These coactivators recruit histone acetyltransferase (HAT), the TRAP/DRIP/mediator complex, the SIRT1 histone deacetylase complex and the SWI/SNF chromatin-remodelling complexes to regulate the epigenetic state of local chromatin [52-55]. At least two non-mutually exclusive mechanisms are engaged by PGC- $1 \alpha$ to regulate rhythmic expression of genes involved in hepatic gluconeogenesis: direct transcriptional regulation and indirect regulation through ubiquitin-specific protease 2 (Usp2). Previous studies have established hepatic nuclear factor $4 \alpha$ (HNF4 $\alpha$ ), glucocorticoid receptor (GR) and forkhead box O1 (FoxO1) as key mediators of gluconeogenic gene regulation by PGC- $1 \alpha$ $[38,56]$. As such, it is probably that cyclic induction of PGC- $1 \alpha$ leads to rhythmic activation of its transcriptional partners and target gene expression.

\section{C $C_{\text {PGC-1 }}$ coactivators directly regulate the core clock and metabolic gene programmes through coactivating specific transcriptional partners. $)$}

Alternatively, PGC- $1 \alpha$ also regulates hepatic glucogenesis indirectly through its induction of Usp2 [57,58]. The deubiquinating enzymes are a family of cysteine proteases that catalyse the removal of ubiquitin from their target proteins. These proteases deubiquitinate a wide array of substrate proteins and regulate diverse biological processes, including 
vesicular trafficking, signal transduction, gene transcription as well as metabolism. In primary hepatocytes, the expression of Usp2 was strongly stimulated by PGC-1 $\alpha$. Further, Usp2 expression exhibits strong diurnal rhythms in several tissues, including the liver and skeletal muscle. It is notable that Usp2 is the only ubiquitin-specific protease exhibiting significant circadian regulation. At the functional level, Usp2 is required for the induction of gluconeogenic and fatty acid $\beta$-oxidation genes by PGC-1 $\alpha$ in hepatocytes. Adenoviral-mediated expression of Usp2 in the liver increases hepatic glucose production and exacerbates glucose intolerance in high-fat diet-induced obese mice, whereas in vivo RNAi knockdown of Usp2 improves systemic glycemic control. Importantly, hepatic rhythmic expression of Usp2 is required for maintaining diurnal glucose regulation in response to restricted feeding. Together, these studies delineate a novel pathway that links hormonal and circadian signals to gluconeogenesis and glucose homeostasis.

Posttranslational mechanisms also play an important role in clock function [59-62]. In particular, casein kinase $1 \delta(\mathrm{CK} 1 \delta)$ and $\mathrm{CK} 1 \varepsilon$ regulate the length of circadian period through phosphorylating several clock proteins. Mutation of $C K 1 \varepsilon$ is responsible for the tau mutant phenotype in Syrian hamsters, which is characterized by shortened circadian period $[63,64]$. Interestingly, mutation of Doubletime, a Drosophila ortholog of $C K 1 \varepsilon$, also results in altered period length [65-67]. A missense mutation (T44A) of $C K 1 \delta$ causes familial advanced sleep-phase syndrome, which is characterized by early sleep onset and early awakening in the morning [68]. Phosphorylation of clock proteins by $\mathrm{CK} 1 \delta$ regulates their stability, subcellular localization and protein-protein interaction. In a search for proteins associated with PGC- $1 \alpha$, it was discovered that both $\mathrm{CK} 1 \delta$ and $\mathrm{CK} 1 \varepsilon$ physically interact with PGC- $1 \alpha$ [69]. PGC- $1 \alpha$ serves as a substrate of CK1 with phosphorylation sites mapped to a domain near the C-terminus of PGC- $1 \alpha$. CK1-mediated phosphorylation reduced PGC- $1 \alpha$ protein stability and transcriptional activity, resulting in impaired hormonal induction of gluconeogenic gene expression and glucose output. These findings illustrate that the clock oscillator reciprocally signals to $P G C-1 \alpha$ and its downstream metabolic gene programmes through transcriptional and posttranslational mechanisms.

\section{Circadian Regulation of Autophagy}

Autophagy is a conserved cellular process responsible for turnover of long-lived proteins and organelles, and plays an essential role in organismal adaptation to starvation $[70,71]$. Nutrient limitation initiates a cascade of vesicle nucleation, autophagosome formation, docking and fusion with lysosome and subsequent lysosomal degradation. Autophagy is markedly induced in neonatal tissues and in adult tissues in response to starvation [72,73]. Defects in autophagy result in lower plasma glucose and amino acid concentrations during the early postnatal period, leading to impaired adaptation and survival. In addition, autophagy is required for removing protein aggregates, damaged organelles and certain pathogens [71]. Autophagy deficiency has been implicated in the pathogenesis of several disease conditions, such as cancer, diabetes, hepatic steatosis, skeletal myopathy and neurodegeneration [74].

Electron microscopy studies performed in the 1970s showed that the number of autophagic vacuoles varies throughout the day in several cell types, including the inner segment of retina rod cells, cardiomyocytes, hepatocytes, pancreatic acinar cells and proximal tubules of kidney in rats $[75,76]$. Using a combination of autophagy markers and flux measurements, recent studies indicated that autophagy activity exhibits a robust diurnal rhythm in the liver, heart and skeletal muscle $[77,78]$. Autophagy flux can be estimated by measuring the degradation rate of microtubule-associated protein 1 light chain 3-II (LC3-II), a marker for autophagy induction. This measurement revealed that autophagy flux in the liver reached a peak in the afternoon and decreased to lower levels in the dark phase. Importantly, the cyclic activation of autophagy flux in the liver is associated with rhythmic expression of autophagy genes [78]. The physiological significance of autophagy cycles remains unknown. It is possible that close coupling of autophagic protein degradation to the body clock may provide unique advantages for mammals to maintain systemic nutrient and energy homeostasis. Further, time-dependent remodelling of cellular proteomes and organelles may be fundamentally important for metabolic homeostasis along the temporal axis. The latter potentially serves an important function in restricting cellular metabolic functions to specific time periods during LD cycles.

Transcriptional regulation of autophagy genes has emerged as an important mechanism in autophagy. Several transcription factors have been identified that regulate various aspects of the autophagy gene programme, including Forkhead transcription factor $\mathrm{O} 3$ (FoxO3) and transcription factor EB (TFEB). FoxO3 was identified as a regulator of autophagy in skeletal myocytes by stimulating the expression of autophagy genes, including LC3B, Gabarapl1, Bnip3 and Bnip3l [79]. TFEB induces the expression of a large number of genes involved in lysosome biogenesis, autophagy and lysosomal exocytosis [80,81]. TFEB is colocalized with mTORC1 under normal growth conditions and undergoes nuclear translocation in response to starvation and lysosomal stress $[82,83]$. In the context of circadian autophagy, CCAAT/enhancer binding protein $\beta(\mathrm{C} / \mathrm{EBP} \beta)$ is emerging as a key regulator of rhythmic autophagy gene expression in the liver [78]. $\mathrm{C} / \mathrm{EBP} \beta$ is a basic leucine zipper transcription factor that regulates diverse biological processes. The expression of $\mathrm{C} / \mathrm{EBP} \beta$ itself is highly rhythmic and is dependent on the liver clock. Gain-of-function studies using adenoviral-mediated gene expression indicated that $\mathrm{C} / \mathrm{EBP} \beta$ promotes the programme of autophagy gene expression and induces autophagic protein degradation in cultured hepatocytes. $\mathrm{C} / \mathrm{EBP} \beta$ directly binds to the promoters of autophagy genes and activates their transcription [78]. $C / E B P \beta$ itself is a target of core clock and is required for the regulation of rhythmic autophagy in the liver.

\footnotetext{
$\mathbf{C}$ Circadian control of autophagy rhythm is mediated by transcriptional control of autophagy gene expression by $\mathrm{C} / \mathrm{EBP} \beta$ and PGC-1 $\beta$. $)$
} 


\section{PGC-1 $\beta$ and Rhythmic Autophagy Regulation}

The physiological role of PGC-1 coactivators in transcriptional regulation of autophagy genes has not been established. In co-immunoprecipitation (co-IP) studies, PGC-1 $\beta$ was found to be physically associated with $\mathrm{C} / \mathrm{EBP} \beta$ in transiently transfected 293T cells (Figure 2A), suggesting that it may function as a transcriptional coactivator for $\mathrm{C} / \mathrm{EBP} \beta$. Importantly, adenoviral-mediated overexpression of $P G C-1 \beta$ stimulated the expression of many $\mathrm{C} / \mathrm{EBP} \beta$-regulated autophagy genes in primary hepatocytes, including FIP200, Ulk1, LC3B, Atg2a, Gabarapl1, Bnip3, Atg16l1 and Atp6v0a2, a subunit of vacuolar proton pump (Figure $2 \mathrm{~B}$ ). PGC-1 $\beta$ also stimulates the expression of $C / E B P \beta$, suggesting that these two factors may form a feed-forward regulatory loop. Recent studies have shown that the mammalian Ulk1-FIP200-Atg13 kinase complex, which is equivalent to the Atg1 complex in yeast, integrates diverse nutrient signals and controls the initiation of autophagy in the cell $[84,85]$. Liver-specific inactivation of FIP200 severely impaired autophagy in hepatocytes and resulted in liver injury [86]. As such, FIP200 and Ulk1 may provide an important link between PGC-1 $\beta$ and the autophagy pathway.

Activation of autophagy enhances the degradation of sequestosome 1 ( $\mathrm{p} 62$ ), an LC3-interacting protein that delivers cargos for lysosomal degradation. Consistently, we found that PGC- $1 \beta$ significantly reduces steady-state p62 levels in transduced hepatocytes (Figure 2C). Similar effects on p62 levels were also observed in the livers from mice transduced with $P G C-1 \beta$ adenovirus (Figure 2D). Together, these studies show that PGC- $1 \beta$ may serve as a coactivator for $\mathrm{C} / \mathrm{EBP} \beta$ to stimulate autophagy gene expression and enhance autophagy activity. As the expression of $P G C-1 \beta$ exhibits diurnal rhythms in the liver, it is likely that the rhythmic induction of $C / E B P \beta$ and $P G C-1 \beta$ may mediate circadian signalling to drive rhythmic activation of autophagy.

\section{Perspectives}

Circadian metabolic rhythms are a core feature of mammalian nutrient and energy homeostasis and have important implications in health and disease. The mechanisms that integrate metabolic functions with circadian timing in tissue are complex and probably involve reciprocal crosstalk between the metabolic regulatory networks and the biological clock. The molecular clock directly regulates the expression of downstream metabolic genes and participates in driving diurnal metabolic cycles. In parallel, the clock oscillator also responds to nutritional and hormonal cues by adjusting the phase and/or amplitude of rhythmic gene expression. Consequently, while the regulatory circuits are somewhat hardwired between the clockwork and the metabolic gene programmes, remarkable flexibility exists to allow fine-tuning of metabolic output from tissues. The ebb-and-flow of tissue metabolism is emerging to represent unique opportunities for therapeutic intervention by restoring the rhythmicity of the body clock and/or targeting therapies to certain phases of the metabolic cycle.
A

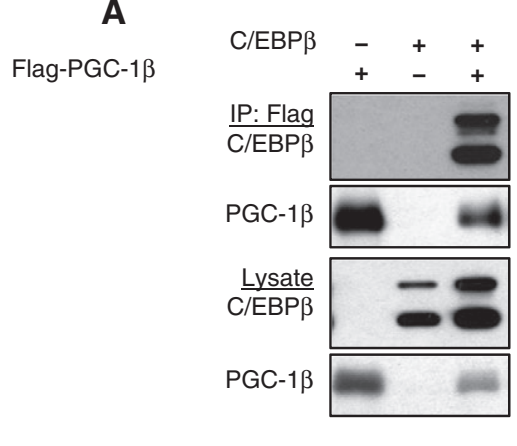

C



B
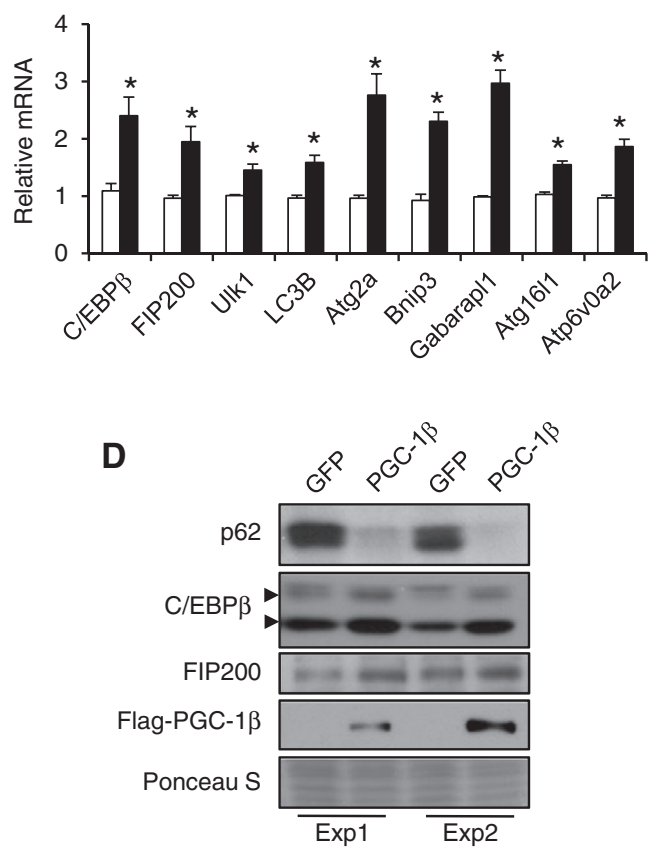

Figure 2. Induction of autophagy by the C/EBP $\beta / \mathrm{PGC}-1 \beta$ transcriptional complex. (A) Physical interaction between PGC-1 $\beta$ and C/EBP $\beta$. (B) qPCR analysis of hepatocytes transduced with green fluorescence protein (GFP) (open) or PGC-1 $\beta$ (filled) adenovirus. Shown is mean \pm standard deviation of one representative experiment in triplicates, ${ }^{*} \mathrm{p}<0.01$. (C) Immunoblots of total lysates from hepatocytes transduced with $G F P$ or $P G C-1 \beta$ adenovirus. (D) Immunoblots of pooled lysates from transduced livers obtained in two independent experiments. C/EBP $\beta$, CCAAT/enhancer binding protein $\beta$; PGC-1, PPAR $\gamma$ coactivator 1; qPCR, quantitative polymerase-chain-reaction. 


\section{Acknowledgements}

The authors would like to thank other members of the laboratory for discussions. This work was supported by the National Institutes of Health (DK077086 and DK095151). The authors apologize to colleagues whose relevant work was not cited here because of space limitation.

\section{Conflict of Interest}

The authors declare no conflict of interest.

\section{References}

1. Damiola F, Le Minh N, Preitner N, Kornmann B, Fleury-Olela F, Schibler U. Restricted feeding uncouples circadian oscillators in peripheral tissues from the central pacemaker in the suprachiasmatic nucleus. Genes Dev 2000; 14 2950-2961.

2. Hara R, Wan K, Wakamatsu H et al. Restricted feeding entrains liver clock without participation of the suprachiasmatic nucleus. Genes Cells 2001; 6 269-278

3. Stokkan KA, Yamazaki S, Tei H, Sakaki Y, Menaker M. Entrainment of the circadian clock in the liver by feeding. Science 2001; 291: 490-493.

4. Geusz ME, Fletcher $C$, Block GD et al. Long-term monitoring of circadian rhythms in c-fos gene expression from suprachiasmatic nucleus cultures. Curr Biol 1997; 7: 758-766.

5. Balsalobre A, Damiola F, Schibler U. A serum shock induces circadian gene expression in mammalian tissue culture cells. Cell 1998; 93: 929-937.

6. Nagoshi E, Saini C, Bauer C, Laroche T, Naef F, Schibler U. Circadian gene expression in individual fibroblasts: cell-autonomous and self-sustained oscillators pass time to daughter cells. Cell 2004; 119: 693-705.

7. Asher G, Sassone-Corsi P. Time for food: the intimate interplay between nutrition, metabolism, and the circadian clock. Cell 2015; 161: 84-92.

8. Asher G, Schibler U. Crosstalk between components of circadian and metabolic cycles in mammals. Cell Metab 2011; 13: 125-137.

9. Bass J, Takahashi JS. Circadian integration of metabolism and energetics. Science 2010; 330: 1349-1354.

10. Dallmann R, Viola AU, Tarokh L, Cajochen C, Brown SA. The human circadian metabolome. Proc Natl Acad Sci U S A 2012; 109: 2625-2629.

11. Eckel-Mahan KL, Patel VR, Mohney RP, Vignola KS, Baldi P, Sassone-Corsi P. Coordination of the transcriptome and metabolome by the circadian clock. Proc Natl Acad Sci U S A 2012; 109: 5541-5546.

12. Kasukawa $T$, Sugimoto $M$, Hida $A$ et al. Human blood metabolite timetable indicates internal body time. Proc Natl Acad Sci U S A 2012; 109: 15036-15041.

13. Lowrey PL, Takahashi JS. Mammalian circadian biology: elucidating genome-wide levels of temporal organization. Annu Rev Genomics Hum Genet 2004; 5: 407-441.

14. Panda S, Antoch MP, Miller BH et al. Coordinated transcription of key pathways in the mouse by the circadian clock. Cell 2002; 109: 307-320.

15. Storch KF, Lipan 0, Leykin I et al. Extensive and divergent circadian gene expression in liver and heart. Nature 2002; 417: 78-83.

16. Ueda HR, Chen W, Adachi A et al. A transcription factor response element for gene expression during circadian night. Nature 2002; 418: 534-539.

17. Zhang $R$, Lahens $N F$, Ballance $H I$, Hughes $M E$, Hogenesch JB. A circadian gene expression atlas in mammals: implications for biology and medicine. Proc Natl Acad Sci U S A 2014; 111: 16219-16224.

18. Copinschi G, Spiegel K, Leproult R, Van Cauter E. Pathophysiology of human circadian rhythms. Novartis Found Symp 2000; 227: 143-157; discussion 157-162..
19. Cutolo $M$, Otsa $K_{1}$ Aakre 0 , Sulli A. Nocturnal hormones and clinical rhythms in rheumatoid arthritis. Ann N Y Acad Sci 2005; 1051: 372-381.

20. Czeisler $C A, K$ Klerman EB. Circadian and sleep-dependent regulation of hormone release in humans. Recent Prog Horm Res 1999; 54: 97-130; discussion 130-132.

21. Fu L, Lee CC. The circadian clock: pacemaker and tumour suppressor. Nat Rev Cancer 2003; 3: 350-361.

22. Jones $C R$, Campbell SS, Zone SE et al. Familial advanced sleep-phase syndrome: a short-period circadian rhythm variant in humans. Nat Med 1999; $\mathbf{5}$ : 1062-1065.

23. Klerman GL, Davidson EM, Kayce MM. Factors influencing the clinical responses of schizophrenic patients to phenothiazine drugs and to placebo. Psychiatr Res Rep Am Psychiatr Assoc 1964; 19: 97-115.

24. Scheer FA, Hilton MF, Mantzoros CS, Shea SA. Adverse metabolic and cardiovascular consequences of circadian misalignment. Proc Natl Acad Sci U S A 2009; 106: 4453-4458.

25. Spiegel K, Tasali E, Leproult R, Van Cauter E. Effects of poor and short sleep on glucose metabolism and obesity risk. Nat Rev Endocrinol 2009; 5: 253-261.


caloric intake prevents metabolic diseases in mice fed a high-fat diet. Cell Metab 2012; 15: 848-860.

27. Turek FW, Joshu C, Kohsaka A et al. Obesity and metabolic syndrome in circadian Clock mutant mice. Science 2005; 308: 1043-1045.

28. Lamia KA, Storch KF, Weitz CJ. Physiological significance of a peripheral tissue circadian clock. Proc Natl Acad Sci U S A 2008; 105: 15172-15177.

29. Rudic RD, McNamara P, Curtis AM et al. BMAL1 and CLOCK, two essential components of the circadian clock, are involved in glucose homeostasis. PLoS Biol 2004; 2: e377.

30. Lee J, Kim MS, Li R et al. Loss of Bmal1 leads to uncoupling and impaired glucose-stimulated insulin secretion in beta-cells. Islets 2011; $\mathbf{3}: 381-388$.

31. Marcheva B, Ramsey KM, Buhr ED et al. Disruption of the clock components CLOCK and BMAL1 leads to hypoinsulinaemia and diabetes. Nature 2010; 466 : 627-631.

32. Bugge $A$, Feng $D$, Everett $\sqcup$ et al. Rev-erbalpha and Rev-erbbeta coordinately protect the circadian clock and normal metabolic function. Genes Dev 2012; 26: 657-667.

33. Cho $H$, Zhao $X$, Hatori $M$ et al. Regulation of circadian behaviour and metabolism by REV-ERB-alpha and REV-ERB-beta. Nature 2012; 485 : 123-127.

34. Lin J, Handschin C, Spiegelman BM. Metabolic control through the PGC-1 family of transcription coactivators. Cell Metab 2005; 1: 361-370.

35. Scarpulla RC, Vega RB, Kelly DP. Transcriptional integration of mitochondrial biogenesis. Trends Endocrinol Metab 2012; 23: 459-466.

36. Puigserver $P, W u$ Z, Park CW, Graves R, Wright M, Spiegelman BM. A cold-inducible coactivator of nuclear receptors linked to adaptive thermogenesis. Cell 1998; 92: 829-839.

37. Herzig $S$, Long $F$, Jhala US et al. CREB regulates hepatic gluconeogenesis through the coactivator PGC-1. Nature 2001; 413: 179-183.

38. Yoon JC, Puigserver $\mathrm{P}$, Chen $\mathrm{G}$ et al. Control of hepatic gluconeogenesis through the transcriptional coactivator PGC-1. Nature 2001; 413: 131-138.

39. Baar $K$, Wende AR, Jones TE et al. Adaptations of skeletal muscle to exercise: rapid increase in the transcriptional coactivator PGC-1. FASEB J 2002; 16: 1879-1886.

40. Terada $S$, Goto M, Kato M, Kawanaka K, Shimokawa $T$, Tabata I. Effects of low-intensity prolonged exercise on PGC-1 mRNA expression in rat epitrochlearis muscle. Biochem Biophys Res Commun 2002; 296: 350-354.

41. Lin J, Yang R, Tarr PT et al. Hyperlipidemic effects of dietary saturated fats mediated through PGC-1 beta coactivation of SREBP. Cell 2005; 120: 261-273. 
42. Lin J, Wu PH, Tarr PT et al. Defects in adaptive energy metabolism with CNS-linked hyperactivity in PGC-1alpha null mice. Cell 2004; 119: 121-135.

43. Lelliott CJ, Medina-Gomez G, Petrovic N et al. Ablation of PGC-1beta results in defective mitochondrial activity, thermogenesis, hepatic function, and cardiac performance. PLoS Biol 2006; 4: e369.

44. Lin J, Wu H, Tarr PT et al. Transcriptional co-activator PGC-1 alpha drives the formation of slow-twitch muscle fibres. Nature 2002; 418: 797-801.

45. Hernandez C, Molusky M, Li Y, Li S, Lin JD. Regulation of hepatic ApoC3 expression by PGC-1beta mediates hypolipidemic effect of nicotinic acid. Cell Metab 2010; 12: 411-419.

46. Ishii KA, Fumoto $T$, Iwai $K$ et al. Coordination of PGC-1beta and iron uptake in mitochondrial biogenesis and osteoclast activation. Nat Med 2009; 15: 259-266.

47. Lin JD, Liu C, Li S. Integration of energy metabolism and the mammalian clock. Cell Cycle 2008; 7: 453-457.

48. Liu C, Li S, Liu T, Borjigin J, Lin JD. Transcriptional coactivator PGC-1alpha integrates the mammalian clock and energy metabolism. Nature 2007; 447 477-481.

49. Alenghat T, Meyers $K_{1}$ Mullican SE et al. Nuclear receptor corepressor and histone deacetylase 3 govern circadian metabolic physiology. Nature 2008; 456: 997-1000.

50. Yin L, Lazar MA. The orphan nuclear receptor Rev-erbalpha recruits the $\mathrm{N}$-CoR/histone deacetylase 3 corepressor to regulate the circadian Bmal1 gene. Mol Endocrinol 2005; 19: 1452-1459.

51. Sonoda J, Mehl IR, Chong LW, Nofsinger RR, Evans RM. PGC-1beta controls mitochondrial metabolism to modulate circadian activity, adaptive thermogenesis, and hepatic steatosis. Proc Natl Acad Sci U S A 2007; 104: 5223-5228.

52. Puigserver $P$, Adelmant $G, W u Z$ et al. Activation of PPARgamma coactivator- 1 through transcription factor docking. Science 1999; 286: 1368-1371.

53. Rodgers JT, Lerin C, Haas W, Gygi SP, Spiegelman BM, Puigserver P. Nutrient control of glucose homeostasis through a complex of PGC-1alpha and SIRT1. Nature 2005; 434: 113-118.

54. Wallberg AE, Yamamura S, Malik S, Spiegelman BM, Roeder RG. Coordination of p300-mediated chromatin remodeling and TRAP/mediator function through coactivator PGC-1alpha. Mol Cell 2003; 12: 1137-1149.

55. Li S, Liu C, Li N et al. Genome-wide coactivation analysis of PGC-1alpha identifies BAF60a as a regulator of hepatic lipid metabolism. Cell Metab 2008; 8: $105-117$.

56. Puigserver $P$, Rhee J, Donovan J et al. Insulin-regulated hepatic gluconeogenesis through F0X01-PGC-1alpha interaction. Nature 2003; 423: 550-555.

57. Molusky MM, Li S, Ma D, Yu L, Lin JD. Ubiquitin-specific protease 2 regulates hepatic gluconeogenesis and diurnal glucose metabolism through 11 beta-hydroxysteroid dehydrogenase 1. Diabetes 2012; 61: 1025-1035.

58. Molusky MM, Ma D, Buelow K, Yin L, Lin JD. Peroxisomal localization and circadian regulation of ubiquitin-specific protease 2. PLoS One 2012; 7: e47970.

59. Reppert SM, Weaver DR. Molecular analysis of mammalian circadian rhythms. Annu Rev Physiol 2001; 63: 647-676.

60. Reppert SM, Weaver DR. Coordination of circadian timing in mammals. Nature 2002; 418: 935-941.

61. Loudon AS, Meng OJ, Maywood ES, Bechtold DA, Boot-Handford RP, Hastings $\mathrm{MH}$. The biology of the circadian $\mathrm{Ck} 1$ epsilon tau mutation in mice and Syrian hamsters: a tale of two species. Cold Spring Harb Symp Quant Biol 2007; 72 261-271.

62. Virshup DM, Eide EJ, Forger DB, Gallego M, Harnish EV. Reversible protein phosphorylation regulates circadian rhythms. Cold Spring Harb Symp Quant Biol 2007; 72: 413-420.

63. Lowrey PL, Shimomura K, Antoch MP et al. Positional syntenic cloning and functional characterization of the mammalian circadian mutation tau. Science 2000; 288: 483-492.
64. Ralph MR, Menaker M. A mutation of the circadian system in golden hamsters. Science 1988; 241 : 1225-1227.

65. Kloss B, Price JL, Saez L et al. The Drosophila clock gene double-time encodes a protein closely related to human casein kinase I epsilon. Cell 1998; 94: 97-107.

66. Price JL, Blau J, Rothenfluh A, Abodeely M, Kloss B, Young MW. Double-time is a novel Drosophila clock gene that regulates PERIOD protein accumulation. Cell 1998; 94: 83-95.

67. Young MW. The molecular control of circadian behavioral rhythms and their entrainment in Drosophila. Annu Rev Biochem 1998; 67: 135-152.

68. Xu Y, Padiath OS, Shapiro RE et al. Functional consequences of a CKI delta mutation causing familial advanced sleep phase syndrome. Nature 2005; 434 : 640-644.

69. Li S, Chen XW, Yu L, Saltiel AR, Lin JD. Circadian metabolic regulation through crosstalk between casein kinase 1 delta and transcriptional coactivator PGC-1alpha. Mol Endocrinol 2011; 25: 2084-2093.

70. He C, Klionsky DJ. Regulation mechanisms and signaling pathways of autophagy. Annu Rev Genet 2009; 43: 67-93.

71. Mizushima N, Komatsu M. Autophagy: renovation of cells and tissues. Cell 2011; 147: $728-741$

72. Kuma $A$, Hatano $M$, Matsui $M$ et al. The role of autophagy during the early neonatal starvation period. Nature 2004 ; 432: 1032-1036.

73. Mizushima N, Yamamoto A, Matsui M, Yoshimori T, Ohsumi Y. In vivo analysis of autophagy in response to nutrient starvation using transgenic mice expressing a fluorescent autophagosome marker. Mol Biol Cell 2004; 15: $1101-1111$.

74. Choi AM, Ryter SW, Levine B. Autophagy in human health and disease. N Engl J Med 2013; 368: 651-662.

75. Pfeifer U, Strauss P. Autophagic vacuoles in heart muscle and liver. A comparative morphometric study including circadian variations in meal-fed rats. J Mol Cell Cardiol 1981; 13: 37-49.

76. Pfeifer U, Scheller H. A morphometric study of cellular autophagy including diurnal variations in kidney tubules of normal rats. J Cell Biol 1975; 64: 608-621.

77. Ma D, Li S, Molusky MM, Lin JD. Circadian autophagy rhythm: a link between clock and metabolism? Trends Endocrinol Metab 2012; 23: 319-325.

78. Ma D, Panda S, Lin JD. Temporal orchestration of circadian autophagy rhythm by C/EBPbeta. EMBO J 2011; 30: 4642-4651.

79. Mammucari C, Milan G, Romanello V et al. Fox03 controls autophagy in skeletal muscle in vivo. Cell Metab 2007; 6: 458-471.

80. Settembre C, Di Malta C, Polito VA et al. TFEB links autophagy to lysosomal biogenesis. Science 2011; 332: 1429-1433.

81. Sardiello M, Palmieri M, di Ronza A et al. A gene network regulating lysosomal biogenesis and function. Science $2009 ; 325$ : 473-477.

82. Pena-Llopis S, Vega-Rubin-de-Celis S, Schwartz JC et al. Regulation of TFEB and V-ATPases by mTORC1. EMBO J 2011; 30: 3242-3258.

83. Settembre C, Zoncu R, Medina DL et al. A lysosome-to-nucleus signalling mechanism senses and regulates the lysosome via mTOR and TFEB. EMBO J 2012; 31: 1095-1108.

84. Hosokawa N, Hara T, Kaizuka T et al. Nutrient-dependent mTORC1 association with the ULK1-Atg13-FIP200 complex required for autophagy. Mol Biol Cell 2009; 20: 1981-1991.

85. Jung $\mathrm{CH}$, Jun $\mathrm{CB}$, Ro $\mathrm{SH}$ et al. ULK-Atg13-FIP200 complexes mediate mTOR signaling to the autophagy machinery. Mol Biol Cell 2009; 20: 1992-2003.

86. Ma D, Molusky MM, Song J et al. Autophagy deficiency by hepatic FIP200 deletion uncouples steatosis from liver injury in NAFLD. Mol Endocrinol 2013; 27: $1643-1654$. 